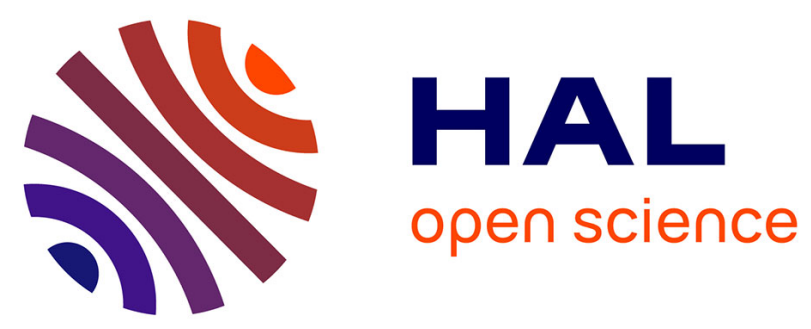

\title{
Can the Partial Peptide SIVSF of $\beta 2$-Adrenergic Receptor Recognize Chirality of Epinephrine Neurotransmitter?
}

Masato Tamura, Tsubasa Sekiguchi, Shun-Ichi Ishiuchi, Anne Zehnacker-Rentien, Masaaki Fujii

\section{To cite this version:}

Masato Tamura, Tsubasa Sekiguchi, Shun-Ichi Ishiuchi, Anne Zehnacker-Rentien, Masaaki Fujii. Can the Partial Peptide SIVSF of $\beta 2$-Adrenergic Receptor Recognize Chirality of Epinephrine Neurotransmitter?. Journal of Physical Chemistry Letters, 2019, 10.1021/acs.jpclett.9b00184 . hal-02070253

\section{HAL Id: hal-02070253 https://hal.science/hal-02070253}

Submitted on 7 Nov 2020

HAL is a multi-disciplinary open access archive for the deposit and dissemination of scientific research documents, whether they are published or not. The documents may come from teaching and research institutions in France or abroad, or from public or private research centers.
L'archive ouverte pluridisciplinaire HAL, est destinée au dépôt et à la diffusion de documents scientifiques de niveau recherche, publiés ou non, émanant des établissements d'enseignement et de recherche français ou étrangers, des laboratoires publics ou privés. 
Can the partial peptide SIVSF of $\beta_{2}$-adrenergic receptor recognize chirality of epinephrine neurotransmitter?

Masato Tamura, ${ }^{1,2}$ Tsubasa Sekiguchi, ${ }^{1,2}$ Shun-ichi Ishiuchi, ${ }^{*, 1,2}$ Anne Zehnacker-Rentien, ${ }^{*, 3,4}$ and Masaaki Fujii*,1,2,4

${ }^{1}$ Laboratory for Chemistry and Life Science, Institute of Innovative Research, Tokyo Institute of Technology, 4259, Nagatsuta-cho, Midori-ku, Yokohama, 226-8503, Japan

${ }^{2}$ School of Life Science and Technology, Tokyo Institute of Technology. 4259 Nagatsuta-cho, Midori-ku, Yokohama, Kanagawa, 226-8503, Japan.

3Institut des Sciences Moléculaires d'Orsay (ISMO), CNRS, Univ. Paris-Sud, Université Paris-Saclay, F-91405 Orsay, France

${ }^{4}$ Tokyo Tech World Research Hub Initiative (WRHI), Institute of Innovation Research, Tokyo Institute of Technology, 4259, Nagatsuta-cho, Midori-ku, Yokohama, Japan

*Corresponding authors, ishiuchi.s.aa@m.titech.ac.jp (SI), anne.zehnacker-rentien@upsud.fr (AZ), mfujii@res.titech.ac.jp (MF),

\begin{abstract}
(148 words)
Chirality plays an essential role in biological molecular recognition such as neurotransmission. Here, we apply electrospray (ESI) - cold ion trap spectroscopy to complexes of a partial binding motif SIVSF of $\beta_{2}$ - adrenergic receptor pocket with $\mathrm{L}$ - and D-epinephrine $\mathrm{AdH}^{+}$. The UV spectrum of the SIVSF-AdH ${ }^{+}$complex is changed drastically when $\mathrm{L}-\mathrm{AdH}^{+}$is replaced by its enantiomer. The isomer-selected IR spectra reveal that $\mathrm{D}-\mathrm{AdH}^{+}$is bound to SIVSF by its protonated amino-group or a single catechol $\mathrm{OH}$, and induces non-helical secondary structures of SIVSF. It is sharp contrast to the helical SIVSF complex with $\mathrm{L}-\mathrm{AdH}+$, which is close to the intrinsic binding structure with two catechol $\mathrm{OH}$ binding in the receptor. It shows that a short pentapeptide SIVSF can distinguish chirality of the ligand $\mathrm{AdH}^{+}$as well as the receptor. This stereoselectivity is suggested to arise from the additional interaction involving the hydroxyl group on the chiral carbon.
\end{abstract}


Molecular chirality is ubiquitous in nature and most of the molecular recognition processes between protein receptors and ligands are characterized by marked enantioselectivity. ${ }^{1-2}$ For example, the perception of an odorant depends on its chirality because it rests on molecular recognition with the so-called G-protein-coupled receptors (GPCR). ${ }^{3}$ Besides their role in taste and smell, GPCR are of particular interest for their role in responses to hormones or in signal transduction, triggered by molecular recognition between agonists and receptors. Binding of an agonist is highly structure, conformation, and enantiomer selective: isoprenaline, a catecholamine agonist of the $\beta_{2}$-adrenergic receptor, shows considerably higher $(x 40) \beta$-stimulating activity for the (-)L enantiomer than the (+) D. ${ }^{1}$ However, data at the molecular level are scarce. $X$-ray crystal structures of active $\beta_{2}$-adrenergic receptors in interaction with their agonist are limited to agonists with natural chirality. ${ }^{4}{ }^{5}$ Computer modelling and site-directed mutagenesis suggest that the interactions involving the $\beta-\mathrm{OH}$ located on the chiral carbon atom of epinephrine could be essential for achieving stereoselectivity. ${ }^{1}$ Recent crystal structures of the agonist-bound $\beta_{2}$-adrenergic receptor reveal that recognition of the ligand involves a limited amino-acid sequence localized on the TM-V helix, namely SIVSF (S : serine, I, Isoleucine, V : Valine, F : phenylalanine). ${ }^{5}$ This sequence acts as the binding site of the catecholamine agonists, with the two serine residues forming hydrogen bonds with the two catechol hydroxyls of the agonist. ${ }^{5}$ These results prompted the gas-phase spectroscopic study of the recognition of the partial $\mathrm{CH}_{3} \mathrm{CO}$-SIVSF$\mathrm{NHCH}_{3}$ peptide of the $\beta_{2}$-adrenergic receptor, shown in Figure 1a, by proper (agonist) or nonproper neurotransmitters, ${ }^{6}$ protonated like at physiological $\mathrm{pH} .{ }^{7}$ Low-temperature gas phase studies allow characterizing molecular recognition without the perturbations due to solvent effects, conformational isomerism, and multiple interaction sites. ${ }^{8-15}$ Moreover, supersonic expansions and cryogenic ion traps have been widely applied to the study of short peptide sequences mimicking segments of larger proteins. ${ }^{16-19}$ By combining a cryogenic ion trap (10K) equipped with an ESI source with conformer-selective laser spectroscopy, we evidenced different binding patterns between SIVSF and proper or non-proper ligands. ${ }^{6}$ While proper ligands, such as protonated L-epinephrine $\left(\mathrm{L}-\mathrm{AdH}^{+}\right.$- see Figure $\left.1 \mathrm{~b}\right)$, form both catechol-bound and amino-bound complexes with SIVSF, only the amino-bound complex is observed for non-proper catecholamines ligands (norepinephrine and dopamine) although both ligands have catechol ring. Moreover, SIVSF adopts an $\alpha$-turn structure, i.e. the shape of the TM-V helix in natural conditions, in the catechol-bound complexes only. From the Xray structural analysis, SIVSF motif is thought to interact with the catechol ring only, however, the results have revealed that SIVSF identify the difference of amine chains. In this work, we interrogate the effect of chirality on the recognition process by comparing the structures of the SIVSF-(L-AdH $\left.{ }^{+}\right)$and SIVSF-(D-AdH $\left.{ }^{+}\right)$complexes. The working hypothesis, 
demonstrated for $\mathrm{L}-\mathrm{AdH}^{+}$, is that the binding motif and the secondary structure of the short peptide sequence reflect the interaction responsible for molecular recognition in the $\beta_{2}$ adrenergic receptor.

Figure 2 shows the UV spectra of the complexes of the SIVSF pentapeptide with a) L$\mathrm{AdH}^{+}$and b) $\mathrm{D}-\mathrm{AdH}^{+}$measured by monitoring the photofragment of $\mathrm{m} / \mathrm{z}=607$. This fragment is assigned to protonated SIVSF, indicating the detachment of neutral epinephrine. Based on the frequency, the observed UV absorption has been assigned to $\pi \pi^{*}$ transition of catechol ring. ${ }^{20-21}$ The UV spectrum of the SIVSF-(L-AdH $\left.{ }^{+}\right)$complex has been reported recently. ref ACIE Two isomers of the SIVSF-(L-AdH $\left.{ }^{+}\right)$complex contribute to this spectrum. The band in the region from $\sim 34600 \mathrm{~cm}^{-1}$ to $\sim 35000 \mathrm{~cm}^{-1}$ indicated by broken lines is assigned to the aminobound complex in which ${\mathrm{L}-A d H^{+}}^{+}$is $\mathrm{H}$-bonded to SIVSF by its protonated amino group (see Figure 2a). The higher frequency band starting from $\sim 35000 \mathrm{~cm}^{-1}$ corresponds to the catechol-bound structure in which L-AdH ${ }^{+}$is $\mathrm{H}$-bonded to SIVSF by its two catechol $\mathrm{OH}$ groups as shown in the figure. A similar UV spectrum, exhibiting bands characteristic of amino- and catechol-bound structures, is observed for the SIVSF complex with isoprenaline, which also has high affinity to $\beta_{2}$-adrenergic receptor. On the other hand, the structured bands above $35000 \mathrm{~cm}^{-1}$ disappear in the UV spectra of the SIVSF complex with norepinephrine and dopamine, which form only amino-bound structures. Thus, the UV spectrum is a good indicator of the binding motif in the SIVSF complex.

The UV spectrum of SIVSF-(D-AdH $\left.{ }^{+}\right)$significantly differs from that of SIVSF-(L-AdH $\left.{ }^{+}\right)$ (Figure 2b). The UV absorption appears at similar frequency as the previously observed amino-bound complexes, and several sharp bands appear strongly in this region. However, the spectral signature of the catechol-bound complex is not clearly observed. These results suggest that SIVSF-(D-AdH ${ }^{+}$) adopts the amino-bound but not the catechol-bound structure. To confirm it, isomer-selected IR spectra were measured.

Isomer-selected IR spectra of the SIVSF complex with L- and D-AdH ${ }^{+}$are measured by IR-UV ion dip spectroscopy (Figure 3). The IR spectra of the complex with L-AdH ${ }^{+}$(Figure $3 a$ and $3 b$ ) are obtained by probing the bands at $34844 \mathrm{~cm}^{-1}$ and $35015 \mathrm{~cm}^{-1}$, which are indicated by arrows and letters in Figure 2a. Different IR spectra directly reflect the structural difference between amino- and catechol-bound complexes. The vibrational bands above $3550 \mathrm{~cm}^{-1}$ in the IR spectrum of the amino-bound complex (Figure 3a) are assigned to the $\mathrm{OH}$ stretching bands in $\mathrm{L}-\mathrm{AdH}^{+}$and that in serine residue in SIVSF. The assignments of the $\mathrm{OH}$ groups are indicated by colors in the IR spectrum and molecular structures shown in the figure. The vibrational frequencies of $\mathrm{OH}$ stretching in the spectrum of SIVSF-(L-AdH $\left.{ }^{+}\right)$are very close to those in $\mathrm{L}_{-} \mathrm{AdH}^{+}$monomer because of its amino-bound structure. These $\mathrm{OH}$ 
stretching bands originating from L-AdH ${ }^{+}$disappear in the IR spectrum of the catechol-bound complex (Figure 3b). It shows that both catechol OH groups are $\mathrm{H}$-bonded to SIVSF and redshifted significantly. Therefore, presence or absence of bands above $3550 \mathrm{~cm}^{-1}$ are the signature of the binding motif in the complex.

Isomer-selected IR spectra of SIVSF-(D-AdH ${ }^{+}$) are measured by probing bands at 34575 $\mathrm{cm}^{-1}, 34585 \mathrm{~cm}^{-1}, 34631 \mathrm{~cm}^{-1}, 34638 \mathrm{~cm}^{-1}$ and $35004 \mathrm{~cm}^{-1}$, which are shown in Figure $3 \mathrm{c}$ $3 \mathrm{~g}$, respectively. The probed UV transitions are indicated by arrows and letters in Figure $2 \mathrm{~b}$. The band at $35004 \mathrm{~cm}^{-1}$ (band $\mathrm{g}$ ) appears in the region of the catechol-bound complex, thus this band may be the signature of the catechol-bound complex. However, the IR spectra in Figure $3 f$ and $3 g$ are the same, thus we assigned band $g$ to a vibronic band of the complex responsible for band $\mathrm{f}$ at $34638 \mathrm{~cm}^{-1}$. Others show different vibrational spectra thus four isomers coexist. Hereafter, we denote the complexes as complex $\mathrm{c}-\mathrm{f}$ according to the probed bands.

We can classify the four isomers into two families by their vibrational spectra. The IR spectra in Figure $3 \mathrm{e}$ and $3 \mathrm{f}$ are similar in the region above $3550 \mathrm{~cm}^{-1}$ : both spectra show free $\beta-\mathrm{OH}$ (shown in green in the figure), intramolecular $\mathrm{H}$-bonded (light blue) and free $\mathrm{OH}$ (orange) in catechol, and $\mathrm{OH}$ in serine (dark blue) at similar positions. This is the same pattern as in amino-bound isomer of SIVSF-(L-AdH $\left.{ }^{+}\right)$. Thus, complexes e and $f$ are aminobound isomers and no $\mathrm{OH}$ bond involving $\mathrm{D}-\mathrm{AdH}^{+}$contributes to the complex formation. The second group comprises complexes $c$ and $d$ because their spectra are clearly different from those of complexes e and f. Particularly, the free $\mathrm{OH}$ stretching in catechol is missing in their spectra while it appears clearly in the former group. Another characteristic point is appearance of broad bands at $\sim 3200 \mathrm{~cm}^{-1}$, which are signature of $\mathrm{H}$-bonded stretching vibration. According to the absence of free $\mathrm{OH}$ of catechol ring, it is natural to assign the broad band to the $\mathrm{OH}$ stretching in catechol. Complexes $\mathrm{c}$ and $\mathrm{d}$ show the intramolecular $\mathrm{H}$ bonded catechol $\mathrm{OH}$ and free $\beta-\mathrm{OH}$ in contrast to the catechol-bound complex with $\mathrm{L}-\mathrm{AdH}^{+}$ (Figure $3 b$ ). From the spectral signature, we conclude that the complexes $c$ and $d$ are partial catechol-bound structure in which only one of $\mathrm{OH}$ bonds interacts with SIVSF and the $\beta-\mathrm{OH}$ remains free.

The spectrum in the range below $3550 \mathrm{~cm}^{-1}$ reflects the $\mathrm{H}$-bonding pattern of the $\mathrm{NH}$ bonds in SIVSF. ${ }^{22-23}$ Thus, the information about the secondary structure can be obtained from the spectra in this range. The spectra of complexes $c$ and $d$, and complexes e and $f$ are similar to each other in this range. Thus, the complexes in each pair have almost the same secondary structure. However the spectral patterns of complexes $\mathrm{c}-\mathrm{f}$ are all different from that of amino- and catechol-bound complexes with L-AdH ${ }^{+}$(Figure $3 a$ and $3 b$ ). Thus, the structures of the peptide are differently modified by binding of $D$-enantiomer. The spectra of 
the amino-bound complexes e and $f$ have relatively better correspondence to the spectrum of the amino-bound isomer with L-AdH+ (Figure 3a) in contrast to those of complexes $\mathrm{c}$ and d.

In summary, the experimental results for SIVSF-(D-AdH $\left.{ }^{+}\right)$indicate four isomers which can be classified into two structural families. One is amino-bound and the secondary structure of the peptide is rather similar to that in the amino-bound complex with $\mathrm{L}-\mathrm{AdH}+$. Another is partial catechol-bound and the secondary structure of the peptide is different from that of others.

Both L- and D-AdH ${ }^{+}$complexes show amino-bound structure, thus this binding motif is insensitive to chirality. For $\mathrm{D}-\mathrm{AdH}^{+}$, the catechol-bound structure observed for $\mathrm{L}-\mathrm{AdH}^{+}$ complexes is not adopted. Instead, the binding of $\mathrm{D}-\mathrm{AdH}^{+}$produces the partial catecholbound complex in which the SIVSF peptide does not take complete helical structure. In this binding structure, only a single catechol $\mathrm{OH}$ is $\mathrm{H}$-bonded to SIVSF, and $\beta-\mathrm{OH}$ is free. This is clear contrast to the complete catechol-bound structure with $\mathrm{L}-\mathrm{AdH}^{+}$, in which even catechol intramolecular $\mathrm{H}$-bonded $\mathrm{OH}$ is forced to form $\mathrm{H}$-bonding to helical SIVSF peptide. The results obtained here also suggest that induced-fit between the peptide and the ligand is essential and that an imperfect SIVSF helical shape does not allow complete but partial catechol binding.

Another characteristic result is presence of free $\beta-\mathrm{OH}$ except for the catechol-bound complex with L-AdH ${ }^{+}$. This means that $\beta-\mathrm{OH}$ is involved in the formation of the catecholbound complex. This suggests the essential contribution of $\mathrm{OH}$ on the chiral $\beta$-carbon for the stereoselectivity of the interaction. In the crystal structure of $\beta_{2}$-adrenergic receptor, the chiral $\mathrm{OH}$ is estimated to make polar contact with D113 and N312. ${ }^{24}$ SIVSF does not include these residues; nevertheless, SIVSF recognizes the chirality of the ligand by providing the third interaction point necessary for chiral recognition. The molecular recognition in the receptor is dynamic, and the ligand moves from inlet to the receptor pocket. The third interaction of SIVSF may be important to stabilize the ligand to the position that observed by the X-ray structural analysis. Further understanding on the nature of the third interaction points requires high-level quantum chemical calculations that will be reported in the future.

\section{Experimental methods}

A cryogenic ion trap equipped with ESI was used for measuring ultraviolet (UV) and conformer-selective infrared (IR) spectra of biomolecular ions by laser photodissociation and double resonance spectroscopy. Details of the experimental methods have been described previously. Briefly, the SIVSF peptide was synthesized by the Fmoc solid-phase method. The SIVSF peptide and $\mathrm{AdH}^{+}$were dissolved in methanol containing $0.5 \%$ formic acid at the 
concentration of $10^{-5} \mathrm{M}$. This solution was electrosprayed and introduced to vacuum through a glass capillary heated to $60{ }^{\circ} \mathrm{C}$. Only the complex ions of the SIVSF peptide with $\mathrm{AdH}^{+}$were guided to a cryogenic quadrupole ion trap (QIT) through mass selection by a quadrupole mass spectrometer and deflection by a quadrupole bender. The QIT was cooled to $4 \mathrm{~K}$ by a closed cycle He refrigerator. He was introduced to the QIT via a pulsed nozzle. Collided by He gas, the ions trapped by the QIT were cooled to $\sim 10 \mathrm{~K}$. Then the ions were irradiated with a tunable UV laser. The photofragments produced after UV absorption were detected by a time-of-flight mass spectrometer (TOF-MS). By monitoring the amplitude of the photofragment signal, the UV photodissociation (UVPD) spectra of the ions were measured. The UVPD corresponds to the UV absorption convoluted by the fragmentation efficiency, which we assume to be constant in the studied frequency range.

The IR spectra were measured by IR-UV ion dip spectroscopy. The frequency of the UV laser was fixed to a certain band in the UV spectrum to excite the isomer associated with the UV band. The amplitude of the photofragment signal is proportional to the amount of the probed isomer in the ground vibrational level. Before UV irradiation, a tunable IR laser was introduced to the trap. If the IR laser vibrationally excites the probed isomer, the amount of the photofragment signal is reduced because of the decrease of the ions in the ground vibrational level. By monitoring this depletion of the photofragment signal, isomer-selected IR spectra were measured.

\section{Acknowledgements}

This work was supported in part by KAKENHI (JP205104008) on innovative area (2503), KAKENHI (JP15H02157, JP15K13620, JP16H06028), World Research Hub Initiatives at the Tokyo Institute of Technology, and the Cooperative Research Program of the "Network Joint Research Center for Materials and Devices" from the Ministry of Education, Culture, Sports, Science and Technology (MEXT), Japan. All the spectra shown in the paper were measured at the Tokyo Institute of Technology. A.Z. acknowledges travel support from the World Research Hub Initiative (WRHI) of Tokyo Institute of Technology.

\section{References}

1. Wieland, K.; Zuurmond, H. M.; Krasel, C.; Ijzerman, A. P.; Lohse, M. J. Involvement of Asn-293 in Stereospecific Agonist Recognition and in Activation of the Beta(2)-Adrenergic Receptor. Proceedings of the National Academy of Sciences of the United States of America 1996, 93 (17), 9276-9281.

2. Fokkens, J.; Klebe, G. A Simple Protocol to Estimate Differences in Protein Binding 
Affinity for Enantiomers without Prior Resolution of Racemates. Angewandte ChemieInternational Edition 2006, 45 (6), 985-989.

3. Brenna, E.; Fuganti, C.; Serra, S. Enantioselective Perception of Chiral Odorants. Tetrahedron-Asymmetry 2003, 14 (1), 1-42.

4. Rosenbaum, D. M.; Zhang, C.; Lyons, J. A.; Holl, R.; Aragao, D.; Arlow, D. H.; Rasmussen, S. G. F.; Choi, H. J.; DeVree, B. T.; Sunahara, R. K.; Chae, P. S.; Gellman, S. H.; Dror, R. O.; Shaw, D. E.; Weis, W. I.; Caffrey, M.; Gmeiner, P.; Kobilka, B. K. Structure and Function of an Irreversible Agonist-Beta(2) Adrenoceptor Complex. Nature 2011, 469 (7329), 236-240.

5. $\quad$ Ring, A. M.; Manglik, A.; Kruse, A. C.; Enos, M. D.; Weis, W. I.; Garcia, K. C.; Kobilka, B. K. Adrenaline-Activated Structure of Beta(2)-Adrenoceptor Stabilized by an Engineered Nanobody. Nature 2013, 502 (7472), 575-+.

6. Sekiguchi, T.; Tamura, M.; Oba, H.; Carcarbal, P.; Lozada-Garcia, R. R.; ZehnackerRentien, A.; Gregoire, G.; Ishiuchi, S.-i.; Fujii, M. Molecular Recognition by a Short Partial Peptide of the Adrenergic Receptor: A Bottom-up Approach. Angewandte ChemieInternational Edition 2018, 57 (20), 5626-5629.

7. Patil, P. N.; Li, C. L.; Kumari, V.; Hieble, J. P. Analysis of Efficacy of Chiral Adrenergic Agonists. Chirality 2008, 20 (3-4), 529-543.

8. Brodbelt, J. S. Probing Molecular Recognition by Mass Spectrometry. International Journal of Mass Spectrometry 2000, 200 (1-3), 57-69.

9. Schalley, C. A. Molecular Recognition and Supramolecular Chemistry in the Gas Phase. Mass Spectrometry Reviews 2001, 20 (5), 253-309.

10. Speranza, M.; Satta, M.; Piccirillo, S.; Rondino, F.; Paladini, A.; Giardini, A.; Filippi, A.; Catone, D. Chiral Recognition by Mass-Resolved Laser Spectroscopy. Mass Spectrometry Reviews 2005, 24 (4), 588-610.

11. Zehnacker, A.; Suhm, M. A. Chirality Recognition between Neutral Molecules in the Gas Phase. Angewandte Chemie-International Edition 2008, 47 (37), 6970-6992.

12. Zehnacker, A. Chirality Effects in Gas-Phase Spectroscopy and Photophysics of Molecular and Ionic Complexes: Contribution of Low and Room Temperature Studies. International Reviews in Physical Chemistry 2014, 33 (2), 151-207.

13. Tao, W. A.; Cooks, R. G. Chiral Analysis by Ms. Analytical Chemistry 2003, 75 (1), $25 \mathrm{~A}-31 \mathrm{~A}$.

14. Zehnacker, A. Chiral Recognition in the Gas Phase. CRC Press Taylor \& Francis Group: Boca Raton, 2010.

15. Klyne, J.; Bouchet, A.; Ishiuchi, S.; Fujii, M.; Schneider, M.; Baldauf, C.; Dopfer, O. Probing Chirality Recognition of Protonated Glutamic Acid Dimers by Gas-Phase Vibrational 
Spectroscopy and First-Principles Simulations. Physical Chemistry Chemical Physics 2018, 20 (45), 28452-28464.

16. Gloaguen, E.; Mons, M. Isolated Neutral Peptides. In Gas-Phase Ir Spectroscopy and Structure of Biological Molecules, Rijs, A. M.; Oomens, J., Eds. 2015; Vol. 364, pp 225270 .

17. Gord, J. R.; Walsh, P. S.; Fisher, B. F.; Gellman, S. H.; Zwier, T. S. Mimicking the First Turn of an Alpha-Helix with an Unnatural Backbone: Conformation-Specific Ir and Uv Spectroscopy of Cyclically Constrained Beta/Gamma-Peptides. Journal of Physical Chemistry B 2014, 118 (28), 8246-8256.

18. Gerhards, M.; Bialach, P.; Martin, T.; Schwing, K. Isolated Peptides in the Gas Phase: Influence of Aggregation and Solvation. Abstracts of Papers of the American Chemical Society 2011, 241.

19. Rizzo, T. R.; Stearns, J. A.; Boyarkin, O. V. Spectroscopic Studies of Cold, Gas-Phase Biomolecular Ions. International Reviews in Physical Chemistry 2009, 28 (3), 481-515.

20. Gerhards, M.; Perl, W.; Schumm, S.; Henrichs, U.; Jacoby, C.; Kleinermanns, K. Structure and Vibrations of Catechol and Catechol-H2o(D2o) in the S0 and S1 State. The Journal of Chemical Physics 1996, 104 (23), 9362-9375.

21. Wako, H.; Ishiuchi, S. I.; Kato, D.; Feraud, G.; Dedonder-Lardeux, C.; Jouvet, C.; Fujii, M. A Conformational Study of Protonated Noradrenaline by Uv-Uv and Ir Dip Double Resonance Laser Spectroscopy Combined with an Electrospray and a Cold Ion Trap Method. Phys Chem Chem Phys 2017, 19 (17), 10777-10785.

22. Schwing, K.; Gerhards, M. Investigations on Isolated Peptides by Combined Ir/Uv Spectroscopy in a Molecular Beam - Structure, Aggregation, Solvation and Molecular Recognition. International Reviews in Physical Chemistry 2016, 35 (4), 569-677.

23. Chin, W.; Piuzzi, F.; Dimicoli, I.; Mons, M. Probing the Competition between Secondary Structures and Local Preferences in Gas Phase Isolated Peptide Backbones. Phys Chem Chem Phys 2006, 8 (9), 1033-48.

24. Ring, A. M.; Manglik, A.; Kruse, A. C.; Enos, M. D.; Weis, W. I.; Garcia, K. C.; Kobilka, B. K. Adrenaline-Activated Structure of Beta2-Adrenoceptor Stabilized by an Engineered Nanobody. Nature 2013, 502 (7472), 575-579. 